\title{
BMJ Open Detailed study protocol for systematic scoping review of research on child marriage, 2000-2019
}

\author{
Margaret E. Greene (D) , ${ }^{1}$ Manahil Siddiqi (i) ${ }^{2}$
}

To cite: Greene ME, Siddiqi M. Detailed study protocol for systematic scoping review of research on child marriage, 2000-2019. BMJ Open 2021;11:e043845. doi:10.1136/ bmjopen-2020-043845

- Prepublication history and additional material for this paper is available online. To view these files, please visit the journal online (http://dx.doi.org/10. 1136/bmjopen-2020-043845).

Received 01 September 2020 Revised 08 February 2021 Accepted 05 March 2021

Check for updates

(C) Author(s) (or their employer(s)) 2021. Re-use permitted under CC BY-NC. No commercial re-use. See rights and permissions. Published by BMJ.

${ }^{1}$ Ringgold 576021, GreeneWorks, Washington, District of Columbia, USA

${ }^{2}$ Department of Health Services, University of Washington School of Public Health, Seattle, Washington, USA

Correspondence to Dr Margaret E. Greene; mgreene@greeneworks.com

\section{ABSTRACT}

Introduction The global development agenda reflects greater attention to ending child marriage and supporting adolescent girls than ever before. Limited understandings of the evidence base on child marriage, however, make it challenging to assess gaps in the literature and inform policy and programming to respond to the needs of adolescent girls. The goal of this project is to systematically identify, evaluate and synthesise the global evidence on child marriage.

Methods and analysis We will include articles with a thematic focus on child marriage from all geographic settings, two decades of research (2000-2019) and in four languages (English, Spanish, French and Portuguese). We will search 18 electronic academic databases (7 in English and 4 each in French, Spanish and Portuguese, with 1 overlapping database) and for the grey literature, conduct targeted hand-searches of organisations engaged in work to prevent child marriage. The databases for studies in English are PubMed, PsychINFO, Embase, CINAHL Plus, Popline, Web of Science and Cochrane Library; for studies in French, the databases will be DialNet, Directory of Open Access Journals, Science Direct and Biblioteca CCG-IBT database; in Spanish, DialNet, La Biblioteca Científica Electrónica en Línea, Red Iberoamericana de Innovación y Conocimiento Científico and Jstor and in Portuguese, Coordenação de Aperfeiçoamento de Pessoal de Nível Superior, Biblioteca Virtual em Saúde, Biblioteca Científica Eletrônica On-line and Biblioteca Digital Brasileira de Teses e Dissertações. We will also review reference lists of select articles and seek input from key authors, field practitioners and participants in international convenings. We will collect and analyse data on publication characteristics, including type of document, institutional affiliation, publication year, language, focus country and region, study objective, specific focus, research method, key findings and recommendations of the material offered for future work. The database searches for publications in English were conducted in January 2020 and we plan to complete the searches in French, Spanish and Portuguese in early 2021. Ethics and dissemination As a systematic review of already-published data, this study does not raise ethical or safety concerns. The authors plan to publish the results of the scoping review in a relevant international journal as well as present the results widely following publication. Building on this foundational work, the authors plan to conduct analyses that make use of the rich data.

Registration details The study design adheres to the Preferred Reporting Items for Systematic Reviews and

\section{Strengths and limitations of this study}

This will be the first review to systematically assess the global scope of research on child marriage.

- This review is exceptionally comprehensive, covering four languages (English, Spanish, Portuguese and French), encompassing a 20 -year period (20002019), and including peer-reviewed and grey literature on all geographies.

- Throughout the research process, we are consulting a wide range of experts in the field and soliciting their feedback.

- This review included only English, French, Portuguese and Spanish. Given the global nature of the practice of child marriage, it is likely that research published in other languages was missed.

- In line with scoping methodology, we did not appraise the quality of individual studies. This review aims to provide a comprehensive review of the evidence by being inclusive of all evidence types.

Meta-Analyses extension for Scoping Reviews. Our protocol was registered with Open Science Framework on 14 January 2020 (https://osf.io/awh8v).

\section{INTRODUCTION}

Child marriage, defined as any formal or informal union before the age of 18 years, is a global health and human rights concern and is associated with a wide range of harmful outcomes. $^{1-3}$ While research priorities in any area need to be periodically updated to advance programmatic and policy needs and support advocacy, this is a particularly critical time for stock-taking in the child marriage field. The global development agenda reflects greater attention to ending child marriage and supporting adolescent girls than ever before. The Sustainable Development Goals and the Global Strategy for Women, Children's and Adolescents' Health call explicitly for an end to child, early and forced marriages and unions. $^{4} 5$ International non-governmental organisations, United Nations agencies and governments and civil society groups across the globe are prioritising investment in child 
marriage prevention. To optimise the substantial political attention to child marriage and build global momentum, it is important to examine the state of the evidence and prioritise future actions.

Previous reviews of the child marriage research have tended to be narrow in scope. Existing systematic reviews address highly specific questions on child marriage interventions and their effectiveness, ${ }^{6-9}$ and drawing conclusions about research gaps and priorities are beyond their scope. Lee-Rife $e t a t^{b}$ focused on low-income countries and found that programmes offering incentives and attempting to empower girls can be effective in preventing child marriage. Kalamar $e t a l^{7}$ focused on lowincome and middle-income countries, and found that interventions with a strong economic component were most powerful in their impact on child marriage. Chae and $\mathrm{Ngo}^{8}$ conducted a slightly more inclusive review, and identified empowerment as the most important strategy for reducing child marriage. The review by Malhotra and Elnakib $^{9}$ restricted its focus to experimental and quasiexperimental studies and found that interventions that support girls' schooling through cash or in-kind transfers show the clearest pattern of success in preventing child marriage. That these similar reviews came to somewhat different conclusions reflects methodological and conceptual differences. A clear and consistent classification of interventions is needed-a naming system for programme components-so that future studies and reviews can use the same categories and meaningful comparison can be drawn.

Narrow inclusion criteria relating to language, study methodology and publication type may also preclude critical insights. For example, the review by Malhotra and Elnakib $^{9}$ focused solely on intervention studies that used quantitative experimental or quasi-experimental design. They examined 8 databases in English only, while our study draws from 17 databases in four languages; and finally, recognising the importance of addressing the socioecological model in efforts to end the practice of child marriage, we include a diverse range of study types on girls, family members, community leaders, lawmakers and others, while their inclusion criteria limited their focus to interventions involving girls directly.

Additionally, the vast majority of evidence reviews relating to child marriage research have focused exclusively on specific regions or countries, ${ }^{10-14}$ and/or specific thematic areas such as health ${ }^{15} 16$ or economic consequences. ${ }^{17}$ While this allows for in-depth understandings of child marriage in specific geographies, this global overview of evidence can uncover unique insights about collective research progress, as well as potential areas of focus. In sum, this review of the evidence on child marriage overall goes well beyond previous studies that focus on interventions, thematic areas or geographies-and we believe that this kind of scoping review forms a foundation for any assessment of programmatic interventions.

In light of these gaps, the goal of this project is to systematically identify, evaluate and synthesise the global evidence on child marriage, with the inclusion of all geographic settings, two decades of research and four languages. Our categorisation of child marriage research into 5 research domains and 24 subdomains, a framework adapted from the outcomes of an Expert Group Meeting on child marriage organised by WHO, also allows for deeper and more nuanced analysis. ${ }^{18}$ In this paper, we describe the methodology of our systematic scoping review in detail.

The study design adheres to the Preferred Reporting Items for Systematic Reviews and Meta-Analyses extension for Scoping Reviews (PRISMA-ScR). Our protocol was registered with Open Science Framework on 14 January 2020 (https://osf.io/awh8v).

\section{METHODS AND ANALYSIS}

We have selected a scoping review approach as the most appropriate technique to systematically identify and map the available evidence on child marriage. Scoping review methodology is particularly useful when examining literature across a vast conceptual and methodological range as is the case with research on child marriage. Unlike systematic reviews or meta-analyses, scoping reviews do not narrow the parameters to controlled trials or require quality assessment. Nonetheless, scoping reviews are methodologically rigorous in their approach to examining the extent, range and nature of research activity in a particular field. ${ }^{1920}$ Consistent with the published guidance, this scoping review will involve a six-stage process: (1) identifying the research questions; (2) identifying relevant literature; (3) selecting studies according to inclusion and exclusion criteria; (4) charting the data; (5) collating, summarising and reporting the results and (6) consulting with stakeholders. ${ }^{19}$

\section{Patient and public involvement}

There was no involvement from patients and/or the public in the design of this research, and no patients or members of the public will be involved in the conduct of the research. We have consulted stakeholders and experts throughout the review process.

\section{Stage 1: identifying the research question}

For this systematic scoping review, the overarching research question is, 'What is the extent, range and nature of published research on child marriage from 2000 to 2019?' We have identified several specific subquestions to guide our analysis of the research evidence on child marriage (table 1).

\section{Stage 2: identifying the relevant literature}

The search process for this systematic scoping review has been rigorous and comprehensive involving a four-step approach to identify relevant studies.

First, since January 2020, we have searched seven electronic academic databases (PubMed, PsycINFO, Embase, CINAHL Plus, Popline, Web of Science and the Cochrane 
Table 1 Research subquestions

\begin{tabular}{|c|c|}
\hline Aspect & Research questions \\
\hline \multicolumn{2}{|l|}{ Descriptive overview of publications } \\
\hline Publication volume and timeline & What is the volume of child marriage studies published by year? \\
\hline Geographic location & $\begin{array}{l}\text { Which regions and countries are addressed in the child marriage } \\
\text { literature? }\end{array}$ \\
\hline Study methodology & What methodologies are used to study child marriage? \\
\hline Socioecological model & $\begin{array}{l}\text { What is the distribution of studies on child marriage according to } \\
\text { the level of the socioecological model (individual, family, community, } \\
\text { institution, policy) they focus on? }\end{array}$ \\
\hline Focus population & $\begin{array}{l}\text { Who are the target populations addressed in the child marriage } \\
\text { literature? }\end{array}$ \\
\hline Recommendations & $\begin{array}{l}\text { What research, programmatic and policy recommendations are } \\
\text { emphasised by the child marriage literature? }\end{array}$ \\
\hline \multicolumn{2}{|l|}{ Overview of research themes } \\
\hline Key research domains and subdomains & $\begin{array}{l}\text { What themes are addressed by the child marriage literature? What } \\
\text { is the distribution of studies on child marriage by broad research } \\
\text { domains (child marriage prevalence and trends; causes and } \\
\text { determinants; child marriage consequences; efforts to support } \\
\text { individuals married as children and beyond) and subdomains? }\end{array}$ \\
\hline Evidence gaps & What are the evidence gaps that remain? \\
\hline
\end{tabular}

Library). In early 2021, we will search 12 specialised databases for French, Spanish and Portuguese articles, specifically (see table 2 for full list).

We have developed a search strategy for each database using a combination of free text and controlled vocabulary in consultation with an informational specialist with extensive experience in systematic reviews. Search terms have included variations of terms such as: "child marriage", "early marriage", "child bride", "married adolescents", “cohabit", "girls", “child”, “adolescent", "youth", "teen" and "bride". See online supplemental materials 1 for detailed search strategy.

The second step in the search will involve targeted hand-searches of 15 key organisations that are known to be engaged in work to prevent child marriage (table 2).

Third, we will search the reference lists of selected articles on emerging themes in the field to further identify relevant articles that were not captured. We will prioritise this step, in particular, for papers published in languages other than English.

Finally, we have and will continue to seek input from researchers and practitioners at various international convenings to provide further review and identify any additional studies. These convenings have so far included a UNICEF convening on child marriage and HIV in 2018 (MS); Girls Not Brides consultations on child marriage in Latin America and the Caribbean in 2019 (MEG); a
UC Berkeley, Promundo and GreeneWorks seminar on men and boys and child marriage in 2019 (MEG); Women Deliver in 2019 (MS); a WHO-UNICEF-UNFPA convening on child marriage research priorities in 2019 (MEG); and a Girls Not Brides webinar on child marriage research progress in 2020 (MEG and MS).

\section{Stage 3: study selection}

We will screen articles in two stages; first by title and abstract, followed by the retrieval and screening of fulltext articles.

In the first stage, we will screen the titles and abstracts of articles. We will include papers if they were published between 2000 and 2019; and their titles or abstracts made explicit mention of "child marriage", "early marriage", "forced marriage", "married adolescents". In the case of publications in Spanish or Portuguese about Latin America and the Caribbean, where informal marriages or unions are so common, we will include topics related to child marriage such as "early pregnancy", "early unions", "divorced or widowed adolescents" or "human trafficking". We will review each title and abstract, marking papers as "include", "exclude" or "maybe". For articles marked "maybe", we will discuss to reach consensus.

We will exclude articles at this stage if they are thematically not relevant to child marriage. For example, so far we have excluded papers that focus on marriage or 
Table 2 Search locations

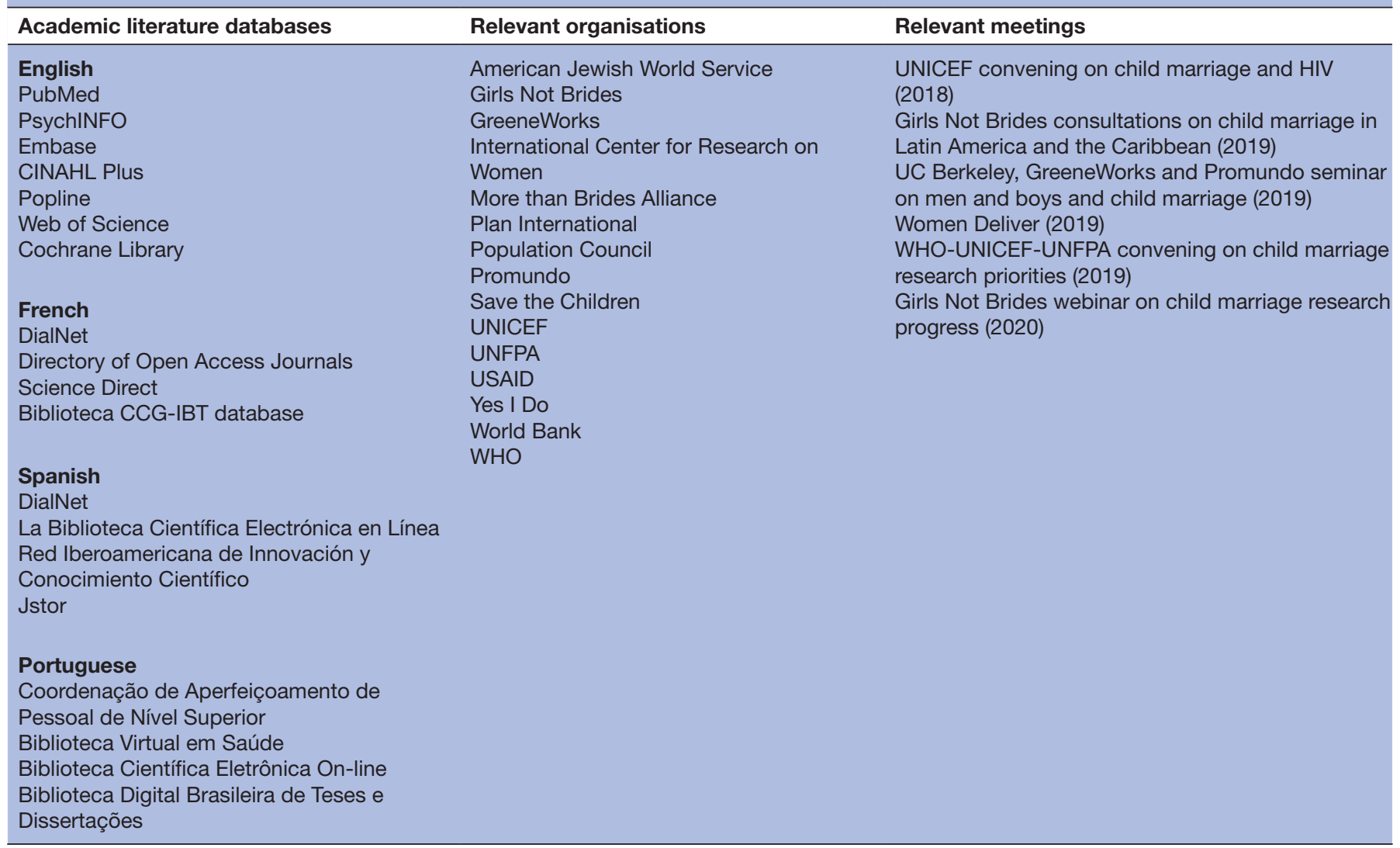

marital life generally, without discussion of adolescents or young people (between the ages of 10 and 19 years) or family, community or institutional-level actors defined by their relationship to the issue of child marriage were excluded. Papers that include both unmarried and married adolescents will be included in this first screening. We will include articles from any geographic setting, research methodology or publication type. The review team has language capacities to review all titles and abstracts in English, French, Spanish or Portuguese. Papers in other languages (eg, Turkish, Farsi or Bahasa Indonesia) that had abstracts in English will be identified but not included in this review.

In the second stage, we will perform a full-text review of the included articles. Reasons for exclusion will be documented and independently reviewed by both principal investigators.

The inclusion criteria we will apply for full-text screenings are below:

- Thematic scope: focus on child, early or forced marriage, or married adolescents.

- Publication year: dated January 2000 through December 2019 (time frame of 20 years).

- Language: English, French, Spanish and Portuguese.

- Geographic focus: any.

- Focus population: we will include research regardless of the sex of the participants.

- Publication type: peer-reviewed or grey literature, evidence-generating or substantively evidence synthesising materials (articles lacking substantive knowledge synthesis, discussion or analysis were excluded).

We will limit the search of the published and grey literature to articles published from 2000 to 2019. The rationale for this time period is to cover the period over which the issue of child marriage came into focus in the international development field. Including the 20-year period will offer the opportunity to observe the increase from the start of this interest to the present.

We will exclude research studies that do not describe the age at marriage or marital status of participants. In assessing informational briefs, we will prioritise the primary literature associated with them.

For all materials, we will limit inclusion to only those that are 'evidence-generating or substantively evidencesynthesising'. If an item lacks substantive knowledge synthesis, discussion or analysis, it will be excluded. We will exclude national strategies and action plans, press releases, news articles, infographics and conference abstracts. When we encounter multiple publications reporting on the same research and including the same complete set of outcomes and knowledge generation, we will include the most comprehensive or the most rigorously produced, favouring a peer-reviewed article over an initial working paper.

The steps for the identification, screening, eligibility and inclusion of studies in the scoping review are detailed in figure 1 . 


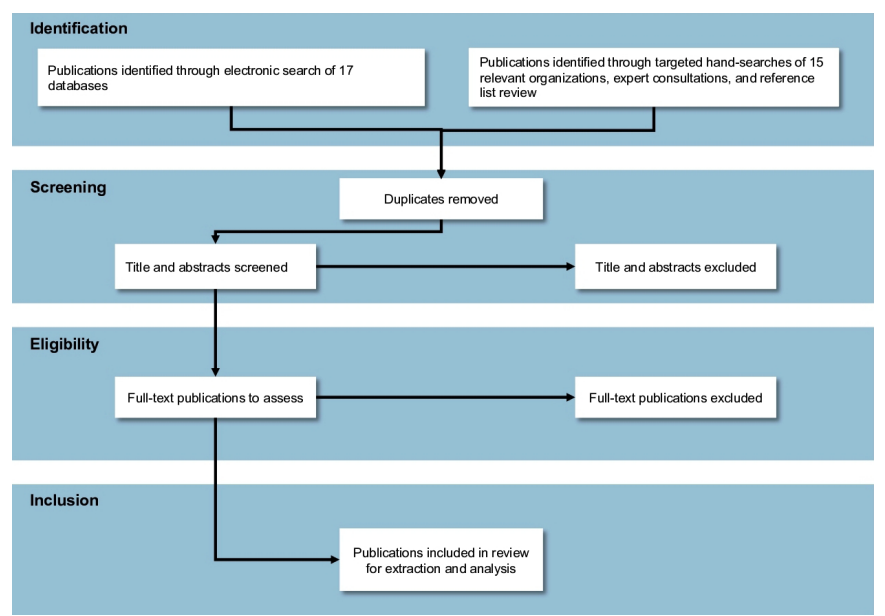

Figure 1 Study selection process.

Records will be screened using Rayyan, a systematic reviews Web app, and managed using Zotero, a reference software.

\section{Stage 4: charting the data}

To confirm study relevance and extract study characteristics, we will develop a standardised data collection tool. The tool will collect data on publication characteristics, including type of document, institutional affiliation, publication year, language, focus country and region, study/publication objective, research method, child marriage research domains and subdomains, key findings and any recommendations for future research/implementation including in the publications.

We have developed the coding framework for the child marriage research category coding based on the results of a WHO expert convening on child marriage research priorities, detailed in the study by Svanemyr et al. ${ }^{18}$ The coding framework covers five broad research categories (prevalence and trends in child marriage, causes and determinants of child marriage, consequences of child marriage, efforts to prevent child marriage and efforts to respond to the needs of married adolescents), along with multiple subcategories described in table 3 .

We will test the data collection tool on a sample of studies to ensure consistency in coding and resolve any issues or ambiguities. Given the high volume of studies eligible for full-text screening, we will engage additional coders to support the data collection process. We will engage coders who have French, Portuguese and/or Spanish language competency, with preference to native speakers. We will make sure to address issues of subjectivity in translation through weekly check-in meetings

Table 3 Coding framework

Broad research domain Subdomains

Prevalence and trends in child 1. Geographic variability in child marriage.

marriage research $\quad 2$. Segmented analyses (religion, ethnicity, education, social class and so on).

3. Age-disaggregated data (particularly for younger adolescent girls, aged 10-14 years).

4. Trends in age at marriage.

Causes and determinants of child marriage
1. Factors contributing to child marriage.

2. Impact of structural factors (eg, urbanisation, migration, climate change and food insecurity, labour markets, civil strife).

3. Norms, perceptions, expectations related to child marriage.

4. Protective factors that prevent child marriage where prevalence is high (positive deviants, public safety and so on).

Consequences of child marriage 1. Maternal, perinatal, sexual and reproductive health.

2. Other health and/or social consequences (mental health, violence, school dropout).

3. Health and social vulnerabilities of younger adolescent girls.

4. Longitudinal data on social, health, development and intergenerational impact of child marriage.

5. Economic costs of child marriage (eg, early childbearing, unintended pregnancy, high fertility, maternal morbidity and mortality, abortion, violence and decreased educational and employment potential).

Efforts to prevent child marriage 1. Essential components of child marriage interventions (eg, required intensity and duration of implementation, mechanisms for delivering these interventions, requirements for scaling up).

2. Efforts to change child marriage norms and practices (eg, cash transfer programmes, school retention programmes).

3. Laws on child marriage and their implementation.

4. Programmatic and policy sanctions and incentives.

5. Lessons from other areas of social and cultural norm change.

6. Indicators, monitoring and evaluation of child marriage prevention, improvement in lives of girls.

7. Policies to prevent child marriage, including health, education and employment opportunities.

Efforts to support individuals married as children
1. Married girls' access to and use of health, education and social services.

2. Married girls' connections to community networks and resources, including peer support.

3. Supporting the development of equitable marital relationships for girls and their husbands.

4. Needs of separated, divorced or widowed girls, and how to respond to them. 
and discussions with each coder to review the data and compare perspectives across translators.

Each coder will conduct an initial test screening of 10 publications that will be assessed by MEG and MS for quality assurance before the coder moves on to further extractions. All papers extracted by coders will be reviewed by MS and MEG to ensure completeness and consistency of extractions.

MS will create a reference guide to maintain consistency across coders, and a chat group for collective discussion of any uncertainties as they arise. For example, when articles are deemed potentially ineligible by the first coder, the article in question will be brought back to the team for discussion and determination of whether to include it or not.

Ineligible abstracted articles will be moved to a separate location in our database to track the stage at which they had been excluded. In line with guidelines for conducting a scoping review, the assessment of methodological quality of the included articles will be performed in a second phase of research.

\section{Stage 5: collating, summarising and reporting the results}

We will generate descriptive statistics using Excel and perform thematic analyses of open-text data. We will present our analysis in diagrammatic and tabular form (numerical summary) as well as in descriptive format (narrative summary). Our analysis will present findings related to publication growth over the 20 -year period with consideration of the transitions of the findings across the areas of child prevalence and trends, causes and determinants, consequences, prevention and support for married adolescents over time. We will also describe shifts in geographical and language patterns, publication types and study methodologies, focus populations and key sectors.

\section{Stage 6: consultation}

Beyond the meetings described in table 2 that have allowed us to consult with experts in the field about relevant literature to include, we will also seize on opportunities to present our methodology and findings to groups of varied experts at various points in our process.

\section{ETHICS AND DISSEMINATION}

As a systematic review of already-published data, this study does not raise ethical or safety concerns. We intend to publish the results of the scoping review in a relevant international journal as well as present the results at public fora following publication. By painting a definitive picture of research on child marriage from 2000 through 2019, this scoping review will offer a richly informed starting point for further efforts to build research, practice and policy in the field and contribute to ending child marriage. Stemming from this foundational work, we plan to conduct two subsequent systematic reviews of the evidence relating to child marriage prevention efforts and programmes to support married girls.

Twitter Margaret E. Greene @Greene_Works and Manahil Siddiqi @ManahilSiddiqi

Acknowledgements The authors would like to thank the reference team that is supporting data extraction including Seung Eun Park and Maya Ulin-0'Keefe, who will work with the authors to organise and prepare the references; Christa Giesecke, who will support searches and full-text extractions in Spanish and Portuguese and Jean-Jacques Kayembe, whose contributions to the searches, screenings and full-text extractions in French will also be essential. The authors would like to thank Catherine Antony, Vaiddehi Bansal, Adriane Berman, Gia Naranjo-Rivera and Christopher Obermeyer for their support on data extractions. The assistance of Sarah Safranek, Health Sciences Librarian, University of Washington has been invaluable. The authors would also like to thank Venkatraman Chandra-Mouli for affirming the importance of this effort.

Contributors MEG conceived the review. MS designed the methodology. MS and MEG have conducted database searches, screened literature and extracted and analysed the data. MEG and MS are supervising the reference team that is conducting data extractions. MS will clean the data and manage the database library.

Funding This research has been funded through the generous support of Kendeda Fund (GreeneWorks 10-18-2017). The Kendeda Fund has strategically invested for nearly a decade in research and programs to end child marriage.

Competing interests None declared.

Patient consent for publication Not required.

Provenance and peer review Not commissioned; externally peer reviewed.

Supplemental material This content has been supplied by the author(s). It has not been vetted by BMJ Publishing Group Limited (BMJ) and may not have been peer-reviewed. Any opinions or recommendations discussed are solely those of the author(s) and are not endorsed by BMJ. BMJ disclaims all liability and responsibility arising from any reliance placed on the content. Where the content includes any translated material, BMJ does not warrant the accuracy and reliability of the translations (including but not limited to local regulations, clinical guidelines, terminology, drug names and drug dosages), and is not responsible for any error and/or omissions arising from translation and adaptation or otherwise.

Open access This is an open access article distributed in accordance with the Creative Commons Attribution Non Commercial (CC BY-NC 4.0) license, which permits others to distribute, remix, adapt, build upon this work non-commercially, and license their derivative works on different terms, provided the original work is properly cited, appropriate credit is given, any changes made indicated, and the use is non-commercial. See: http://creativecommons.org/licenses/by-nc/4.0/.

ORCID iDs

Margaret E. Greene http://orcid.org/0000-0002-9370-5506

Manahil Siddiqi http://orcid.org/0000-0002-2477-3310

\section{REFERENCES}

1 Ganchimeg T, Ota E, Morisaki N, et al. Pregnancy and childbirth outcomes among adolescent mothers: a world Health organization multicountry study. BJOG 2014;121 Suppl 1:40-8.

2 Raj A. When the mother is a child: the impact of child marriage on the health and human rights of girls. Arch Dis Child 2010;95:931-5.

3 Nguyen MC, Wodon Q. Impact of child marriage on literacy and education attainment in Africa. Washington, DC: UNICEF and UNESCO Statistics, 2014.

4 UN General Assembly. Transforming our world: the 2030 agenda for sustainable development, A/RES/70/1. New York: United Nations, 2015.

5 Every Woman Every Child. The global strategy for women's, children's and adolescent's health (2016-2030, 2015.

6 Lee-Rife S, Malhotra A, Warner A, et al. What works to prevent child marriage: a review of the evidence. Stud Fam Plann 2012;43:287-303.

7 Kalamar AM, Lee-Rife S, Hindin MJ. Interventions to prevent child marriage among young people in low- and middle-income countries: a systematic review of the published and gray literature. $J$ Adolesc Health 2016;59:S16-21.

8 Chae S, Ngo T. The global state of evidence on interventions to prevent child marriage." GIRL Center Research Brief No.1. New York: Population Council, 2017. 
9 Malhotra A, Elnakib S. 20 Years of the Evidence Base on What Works to Prevent Child Marriage: A Systematic Review. J Adolesc Health 2021. doi:10.1016/j.jadohealth.2020.11.017. [Epub ahead of print: 11 Jan 2021].

10 ONU Mujeres, United Nations Population Fund, United Nations Children's Fund. A commitment to end child marriage and early unions in Latin America and the Caribbean, UNICEF, 2018.

11 Hodgkinson K, Koster W, Miedema E. Understanding and addressing child marriage: a scoping study of available academic and programmatic literature for the her choice alliance, 2016.

12 Zaman M, Koski A. Child marriage in Canada: a systematic review. PLoS One 2020;15:e0229676.

13 WiLDAF Ghana. Scoping study for parliamentary advocacy programme on Combatting early and forced marriage in Ghana. Parliamentarians for Global Action, 2014.

14 United Nations Children's Fund and United Nations Population Fund. Child marriage in South Asia: an evidence review. Kathmandu: UNICEF, 2019.
15 Irani M, Latifnejad Roudsari R. Reproductive and sexual health consequences of child marriage: a review of literature. J Midwifery Reproductive Health 2019;7:1584-90.

16 Marphatia AA, Ambale GS, Reid AM. Women's marriage age matters for public health: a review of the broader health and social implications in South Asia. Front Public Health 2017;5:269.

17 Parsons J, Edmeades J, Kes A, et al. Economic impacts of child marriage: a review of the literature. Rev Faith Int Aff 2015;13:12-22.

18 Svanemyr J, Chandra-Mouli V, Raj A, et al. Research priorities on ending child marriage and supporting married girls. Reprod Health 2015:12:80.

19 Pham MT, Rajić A, Greig JD, et al. A scoping review of scoping reviews: advancing the approach and enhancing the consistency. Res Synth Methods 2014;5:371-85.

20 Arksey H, O'Malley L. Scoping studies: towards a methodological framework. Int J Soc Res Methodol 2005;8:19-32. 